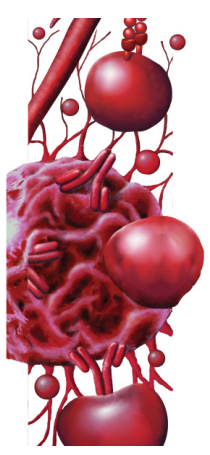

\title{
Targeting the T-cell co-stimulatory CD27/CD70 pathway in cancer immunotherapy: rationale and potential
}

In 2013, cancer immunotherapy was named 'breakthrough of the year' based on the outcome of clinical trials with blocking antibodies to the T-cell co-inhibitory receptors CTLA-4 and PD-1. This success has emphasized that cytotoxic T-cell responses to cancer can occur, but are limited by peripheral tolerance and by immunosuppression in the tumor microenvironment. Targeting of CTLA-4, PD-1 or its ligands partly overcomes these limitations and can now be applied in multiple immunogenic cancer types. Furthermore, an increased success rate is expected from combining CTLA-4 and/or PD-1 blocking with deliberate engagement of T-cell co-stimulatory receptors, particularly TNF receptor ( $R$ ) family members. The TNFR family includes CD27 (Tnfrsf7), for which an agonistic antibody has recently entered clinical trials. In this review, we describe how CD27 co-stimulation impacts the T-cell response, with the purpose to illuminate how CD27 agonism can be exploited in cancer immunotherapy.

Keywords: antibody $\bullet$ cancer $\bullet$ co-inhibition $\bullet$ co-stimulation $\bullet$ cytotoxic T cell

- immunotherapy • ipilimumab • nivolumab • TNF receptor family

\section{The promise of cancer immunotherapy}

The aim of cancer immunotherapy is to elicit a cytotoxic T-lymphocyte (CTL) response that eliminates all tumor cells, regardless of metastatic spread. One key approach is the use of so-called 'immunomodulatory' monoclonal antibodies (mAbs). Such mAbs do not directly target the tumor cells, but are directed at membrane receptors or ligands that regulate the T-cell response. Therapy with these mAbs aims to generate new tumor-specific CTLs from naive $\mathrm{CD}^{+} \mathrm{T}$ cells and to reactivate potentially pre-existing tumor-specific CTLs. The currently successful $\mathrm{mAbs}$ are meant to do this by blocking the interaction of the T-cell co-inhibitory receptors CTLA-4 and PD-1 with their respective ligands. Ipilimumab, which targets CTLA-4, was the first immunomodulatory $\mathrm{mAb}$ to be FDA approved, in this case for the treatment of patients with unresectable or metastatic melanoma [1]. In 2013, Wolchok et al. [2] reported their results from a clinical study in which stage IV melanoma patients were treated with ipilimumab in combination with nivolumab, a blocking $\mathrm{mAb}$ directed at PD-1 [3]. Response rates to monotherapy with ipilimumab or nivolumab were $10.9 \%$ [1] and $28 \%$ [3] respectively, but the response rate to combined therapy was $53 \%$ with a tumor reduction of more than $80 \%$ [2]. Inspired by this result, the journal Science proclaimed cancer immunotherapy as 'Breakthrough of the year 2013' [4].

The challenge is now to increase success rates of this immunotherapeutic approach and to extend it to other cancer types. To do this in a rational manner, the three major obstacles in raising T-cell immunity to cancer should be taken into account. These are: first, central tolerance, that is, deletion of self-reactive $\mathrm{T}$ cells during their development in the thymus. Since tumors are derived from our own cells, the available tumor-specific T-cell repertoire is limited and generally of low affinity. Second, peripheral tolerance. Tumors generally do not send out signals to
Koen van de Ven ${ }^{1}$ \& Jannie Borst*,1 'Division of Immunology, The Netherlands Cancer Institute, Plesmanlaan 121, 1066 CX Amsterdam, The Netherlands

*Author for correspondence:

Tel.: +31205122056

Fax: +3120512 2057

j.borst@nki.nl 
activate dendritic cells (DCs) and without DC activation, the T-cell response will not be initiated. Third, cancer-associated immune suppression. The tumor cells, in dialogue with other (immune) cells in the tumor, create an immunosuppressive environment that counteracts CTL activity [5] (Figure 1).

Given the existence of central tolerance, the tumor types most eligible for immunotherapy are those carrying foreign antigens, such as virus-derived proteins and tumors with a high mutation load. Mutations potentially give rise to altered self-peptides (neoantigens) that can elicit a T-cell response. Efficacy of CTLA- 4 and PD-1 blocking was demonstrated in melanoma and lung cancer that have a high mutation load [6] and are, therefore, expected to present neoantigens. Tumors may also present other antigens to which central tolerance is incomplete, such as cancer-testis antigens.

In immunogenic cancers, T-cell targeting immunotherapy should be aimed at overruling peripheral tolerance to tumor antigens and overruling cancer-associated immune suppression in a complementary fashion (Figure 1). Recent data indicate that the combined effect of CTLA-4 and PD-1 blocking is indeed due to such complementarity. Anti-CTLA- 4 mAb seems to overrule peripheral tolerance, inducing a new T-cell response against the tumor $[7]$. Blocking PD-1 or its ligand PD-L1 seems to primarily overrule the suppression exerted on pre-existing tumor-specific CTLs [8,9]. However, PD-1, like CTLA-4 can also promote peripheral tolerance. Blocking these receptors comes at a cost, since it causes autoimmune symptoms that may be severe, but are generally manageable $[1-3]$.

Apart from blocking co-inhibitory receptors, promoting T-cell responsiveness by engaging co-stimulatory receptors is very attractive. Work in mouse models and other mechanistic studies indicate that these approaches may act complementary and may thus increase therapeutic efficacy, possibly with less autoimmune toxicity when using the right combinations. Agonistic mAbs to a number of co-stimulatory receptors are already in clinical trials [10]. The targets are all members of the TNFR family and include CD27 (Tnfrsf7). The purpose of this review is to outline the mechanism of action of CD27 and its ligand CD70 (Tnfsf7, CD27L) and to predict, based on this information, in which immunotherapeutic strategies CD27 agonism may be exploited to the highest benefit.

Here, we will first review the importance of co-stimulation for the T cell response. Next, we will zoom in on CD27/CD70 and their mechanism of action. In the section 'Conclusion and future perspective,' we will place the reviewed knowledge in the therapeutic context.

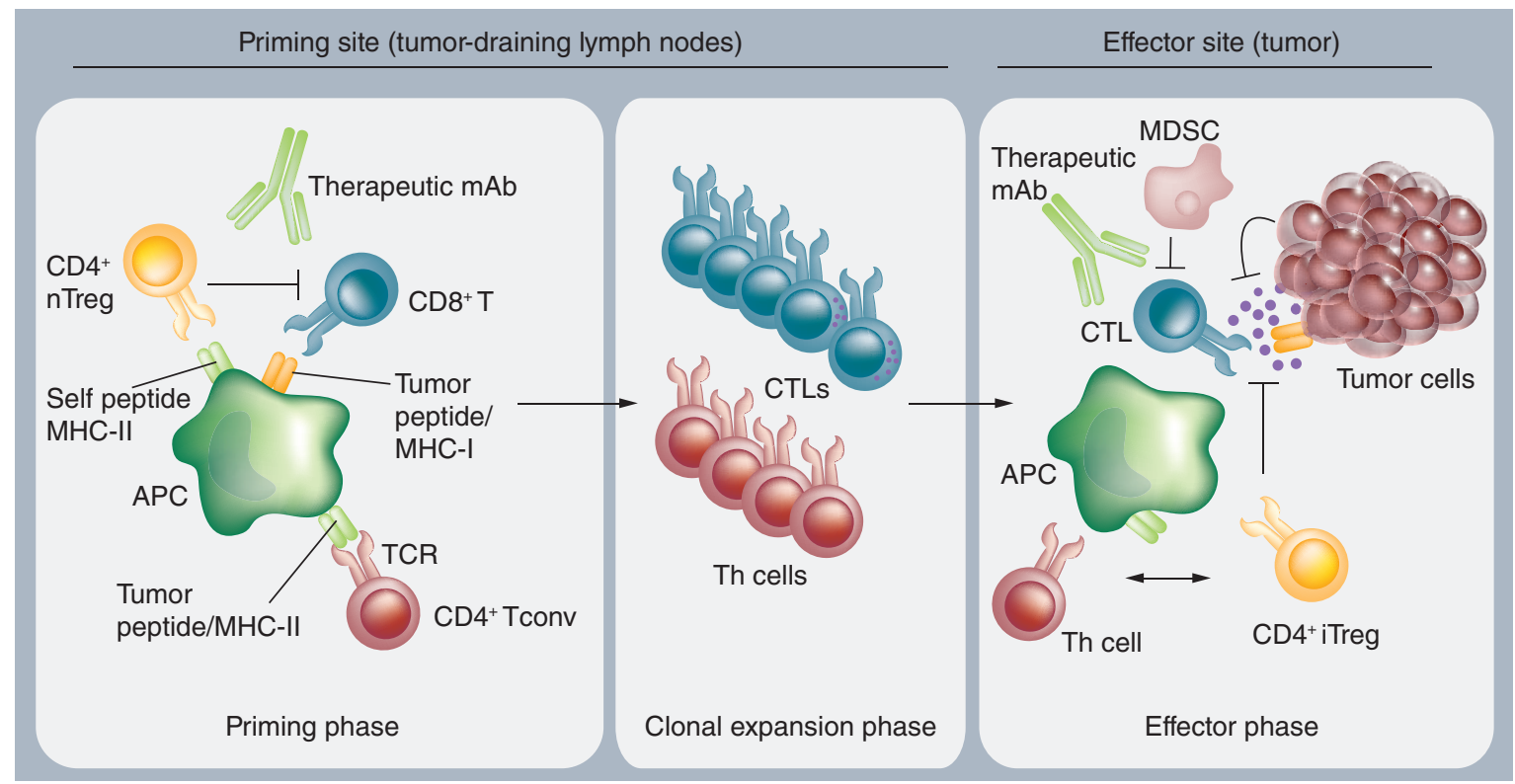

Figure 1. Different phases of the T-cell response to tumor antigen. The scheme shows the priming of conventional $\mathrm{CD}^{+}$and $\mathrm{CD}^{+} \mathrm{T}$ cells in the lymph nodes after their TCR recognizes tumor peptide in the context of, respectively, MHC class I or MHC class II complex on a professional APC. The nTreg inhibits the response of Tconv and CD8 ${ }^{+}$ $T$ cells to self peptides. Therapeutic mAb to CTLA-4 acts on this bottleneck in the T-cell response. In the tumor (the effector site), CTLs can recognize the tumor cells and kill them. iTregs that arise from CD4+ Tconv can inhibit this. Likewise, MDSCs and the tumor cells themselves can inhibit CTL activity. Therapeutic mAb to PD-1 or its ligand acts on the latter bottleneck in the CTL response.

APC: Antigen-presenting cell; CTL: Cytotoxic T lymphocyte; iTreg: Induced regulatory T cell; mAb: Monoclonal antibody; MDSC: Myeloid-derived suppressor cell; nTreg: Natural regulatory T cell; Tconv: Conventional CD4+ T cell; TCR: T-cell receptor. 


\section{Requirements for the induction of a T-cell response}

Dendritic cells (DCs) and other professional antigenpresenting cells (APCs) present self- and foreign peptides (antigens) at their cell surface in the context of major histocompatibility complex (MHC) molecules. Individual $\mathrm{T}$ cells may recognize such peptide/MHC complexes, depending on the specificity of their T-cell antigen receptor (TCR). Whether a T-cell response occurs is dictated by triggering of the TCR (Figure 1). However, for full activation, clonal expansion and concomitant effector differentiation, the $\mathrm{T}$ cell requires co-stimulation. Costimulatory receptors only produce a T-cell response in concert with TCR signaling, thus ensuring antigenspecificity of the response. The two most important co-stimulatory receptors for T-cell priming are CD28 and CD27. CD28 is a member of the immunoglobulin (Ig) family and serves largely as a signal amplifier of the TCR [11]. CD27 is a member of TNF receptor family and has a different mechanism of action, as will be discussed. The activation state of the DC (or other APC) determines whether co-stimulatory receptors are triggered. A resting DC will present antigen, but this will maintain T-cell tolerance since insufficient co-stimulatory signals are present [12]. Only an activated DC is equipped to induce T-cell priming, because it has a higher level of costimulatory molecules at the cell surface. These include the CD28 ligands CD80 and CD86, the CD27 ligand CD70, as well as other TNF ligands. Activated DCs not only contribute to $\mathrm{T}$-cell priming by offering co-stimulatory molecules, but they also make cytokines that shape the effector differentiation programs of $\mathrm{CD}^{+}$and $\mathrm{CD} 8^{+}$ T cells $[12,13]$.

DCs can be activated by pathogen-associated molecular patterns (PAMPs) that trigger pattern recognition receptors (PRR). PAMPs are hallmarks of foreign invaders, such as bacteria and viruses. However, in absence of PAMPs, DCs can still be activated by cognate interaction with $\mathrm{CD}^{+} \mathrm{T}$ cells [13]. When conventional $\mathrm{CD}^{+} \mathrm{T}$ cells recognize peptide/MHC class II complexes on DCs, the DCs gain expression of CD70 and other TNF ligands and upregulate CD80 and CD86 [14-17]. Conversely, CD4+ natural (n) regulatory $\mathrm{T}$ cells (Treg) help to maintain peripheral tolerance. These nTreg have autoreactive TCRs and continuously interact with resting DCs (Figure 1). They constitutively express the co-inhibitory receptors CTLA- 4 and PD-1 that are important for their suppressive activity. CTLA- 4 binds CD80/86 and competes with CD28 for these ligands $[18,19]$. Thus, nTreg can help to prevent the priming of conventional $\mathrm{CD}_{4}^{+}$and $\mathrm{CD}^{+} \mathrm{T}$ cells at least in part by disallowing CD28 engagement.

The T-cell response comprises priming, effector, memory and recall phases, wherein the location of
$T$ cells, their proliferation state and functional activity differ dramatically. Co-stimulatory TNF ligands and their receptors are tuned to these dynamics in expression and function. Antigen is an important factor in driving the expression of TNF ligands and receptors and many of them, therefore, come into play exclusively after immune activation.

\section{TNF (receptor) family members in the T-cell response}

The TNFR family comprises 29 members that are transmembrane proteins with one or more cysteine-rich extracellular domains. These domains form the binding site for the TNF ligands, which are homotrimeric transmembrane proteins [20]. Receptor-ligand interaction thus generally takes place during cell-to-cell communication. Unlike certain other TNFR family members, CD27 and related co-stimulatory TNFRs do not have a proapoptotic death domain. Instead, they have cytoplasmic tail motifs that bind to TNFR associated factors (TRAFs), which are adaptor molecules that link to the NF- $\kappa \mathrm{B}$ and JNK signaling pathways [21]. The NF- $\kappa \mathrm{B}$ pathway counteracts apoptosis and hence, survival signaling is an important shared property of TRAF-binding TNFRs. However, they also have other functions such as the induction of cytokines and chemokines [21]. A number of TNFRs are expressed on T cells and directly influence the T-cell response. This is, for example, the case for CD27, OX40 (Tnfrsf4, CD134), 4-1BB (Tnfrsf9, CD137), CD30 (Tnfrsf8), HVEM (Tnfrsf14) and GITR (Tnfrsf18). However, TNFRs can also indirectly influence the T-cell response by activating APCs, by inducing inflammation and innate immunity, or by organizing lymphoid tissue [21,22].

Co-stimulatory TNFRs support the T-cell response throughout by making their contributions in a complementary fashion. Their complementarity lies largely in the differential timing and location of receptor and ligand expression, rather than in differential signaling [22]. The TNF ligands may have signaling functions as well, but the associated mechanisms are diverse and ill defined. In general, T-cell co-stimulatory TNFRs come into play when $\mathrm{T}$ cells are activated and are exposed to the stress of rapid proliferation and the relocation to nonlymphoid tissues. One of their key functions is to keep activated $\mathrm{T}$ cells alive under these circumstances, but they also orchestrate cell-cell interactions that indirectly support cell survival and function.

\section{Contribution of CD27/CD70 to T-cell activation \& clonal expansion}

In human, CD27 is exclusively expressed in the lymphoid lineage, in other words, by T, B and NK cells and their immediate precursors. In the mouse, CD27 
is additionally expressed on early hematopoietic precursors [23-25]. Importantly, CD27 is already present on naive $\mathrm{CD}^{+}$and $\mathrm{CD}^{+} \mathrm{T}$ cells. Most other costimulatory TNFRs are only synthesized after T cell activation. Upon activation, naive $\mathrm{CD}^{+}$and $\mathrm{CD}^{+}$ $\mathrm{T}$ cells transiently upregulate the expression of CD27. Activated T cells proteolytically shed CD27 from the cell surface, thereby giving rise to circulating soluble $\mathrm{CD} 27$ that can serve as a diagnostic marker of T-cell activation [25]. Terminally differentiated effector $\mathrm{CD} 8^{+}$ $\mathrm{T}$ cells loose CD27, while long-lived central memory cells retain it [23].

CD70 is constitutively expressed on medullary thymic epithelial cells (mTECs) in human and mouse $[15,26,27]$ and on certain unconventional APCs in the mouse intestine [28]. Apart from that, CD70 is exclusively expressed after immune activation. It is found on activated DCs [15-17,29,30], B cells [25], conventional- and regulatory $\mathrm{T}$ cells $[15,24,25,31]$ and NK cells $[27]$. CD70 expression is highly regulated by antigen, since it is under control of PRRs, T cell and B cell antigen receptors and its expression is further tuned by cytokines such as IL-1 $\alpha$, IL-12, TNF $\alpha$, prostaglandin $\mathrm{E}_{2}$ and by CD28- and CD40 co-stimulation [15-17,24,25,29-31]. Epithelial cells and other cell types may acquire CD70 upon malignant transformation [24].

The CD27/CD70 co-stimulatory system plays an important role in immune regulation. At an early stage, human CD27 was recognized as a T-cell costimulatory receptor using in vitro assays with anti$\mathrm{CD} 27 \mathrm{mAb}$ [32]. Upon its cloning, the CD27 ligand CD70 was likewise shown to promote TCR/CD3induced proliferation of naïve $\mathrm{CD} 4^{+}$and $\mathrm{CD}^{+}$human $\mathrm{T}$ cells and the generation of effector cells [33]. The costimulatory function of $\mathrm{CD} 27$ was subsequently solidified by studies in $\mathrm{CD} 27^{-/-}$mice [34] and by treating wild-type mice with CD70 blocking $\mathrm{mAb}$ or recombinant soluble CD70 in infection- and immunization models [35,36].

Studies in mice also elucidated the division of labor between CD27 and CD28 in regulation of the T-cell response. Using mice that were either single- or double deficient for CD27 and CD28, it was revealed that CD27 and CD28 promote clonal expansion of recently activated $\mathrm{T}$ cells in a complementary fashion, apparently acting by distinct mechanisms. CD28 signaling affects expression of cell cycle regulatory proteins and thereby drives activated $\mathrm{T}$ cells into the cell cycle [11]. CD27 signaling does not do so [37-40], but nevertheless increments the number of $\mathrm{CD}^{+}$and $\mathrm{CD}^{+}$ $\mathrm{T}$ cells that enter into the response and become effector cells [34-40]. It also broadens the TCR repertoire of responder $\mathrm{T}$ cells, suggesting that it helps $\mathrm{T}$ cells with a low affinity TCR to enter into the cell cycle [41]. This contribution of CD27 co-stimulation to cell cycle entry and clonal expansion of activated $\mathrm{T}$ cells is based on several distinct mechanisms (Figure 2). In the first place, CD27 co-stimulation counteracts apoptosis in activated $\mathrm{T}$ cells throughout their clonal expansion $[37,42-44]$. It does so by increasing expression of the antiapoptotic molecule Bcl-xL, as has been shown in human and murine activated $\mathrm{CD} 8^{+} \mathrm{T}$ cells $[42,43]$. Furthermore, CD27 co-stimulation downregulates the expression of FasL on $\mathrm{CD}^{+} \mathrm{T}$ cells and decreases the sensitivity of $\mathrm{CD}^{+} \mathrm{T}$ cells to FasL-induced apoptosis [44]. CD27 signaling also supports T-cell activation and clonal expansion by effects on cell metabolism. In $\mathrm{CD}^{+} \mathrm{T}$ cells, it rapidly induces expression of the serine/threonine kinase Pim-1 upon their activation [42]. Pim-1 counteracts apoptosis, but also promotes aerobic glycolysis and protein translation [45], which is very important for rapidly dividing cells. In these ways, CD27/CD70 co-stimulation supports clonal expansion of activated $\mathrm{CD} 8^{+} \mathrm{T}$ cells in an IL-2-independent fashion $[38,42,46]$. CD27 co-stimulation also induces expression of the chemokines CXCL10 and XCL1 in murine and human $\mathrm{CD} 8^{+} \mathrm{T}$ cells. These chemokines most likely promote communication of $\mathrm{T}$ cells with DCs at the site of priming, which indirectly contributes to effector T cell generation [39].

Use of mice with constitutive, transgenic expression of CD70 in DCs has highlighted the potential of CD27 co-stimulation to promote T-cell priming. Upon immunization with MHC class I-restricted peptide in absence of adjuvant, CD70 transgenic DCs allowed generation of robust CTL responses that could eliminate subcutaneously implanted B16 melanoma cells [47]. Ordinarily, such peptide immunization fails to activate DCs and induces T-cell tolerance. Also, when CD70 was present on resting DCs that expressed MHC class I-restricted viral peptides, tolerance was overcome and an effective antiviral CTL response was raised [47]. In the model used, tolerance was defined as CTLA-4- and PD-1-dependent [48], suggesting that CD27/CD70 co-stimulation overruled the inhibitory action of these receptors. As with DCs, transgenic expression of $\mathrm{CD} 70$ on $\mathrm{B}$ cells was also able to promote an antitumor $\mathrm{CD}^{+} \mathrm{T}$ cell response [49].

\section{Contribution of CD27/CD70 to effector \& memory T-cell differentiation}

Activated $T$ cells not only increase in number by clonal expansion, but also acquire specific effector functions. Effector differentiation of $\mathrm{CD}^{+}$and $\mathrm{CD}^{+} \mathrm{T}$ cells is instructed during the priming phase when the $\mathrm{T}$ cells interact with DCs. DCs contribute to effector T-cell differentiation by delivering cytokines and co-stimulatory signals. CD27/CD70 interactions affect $\mathrm{CD}^{+}{ }^{+}$ 


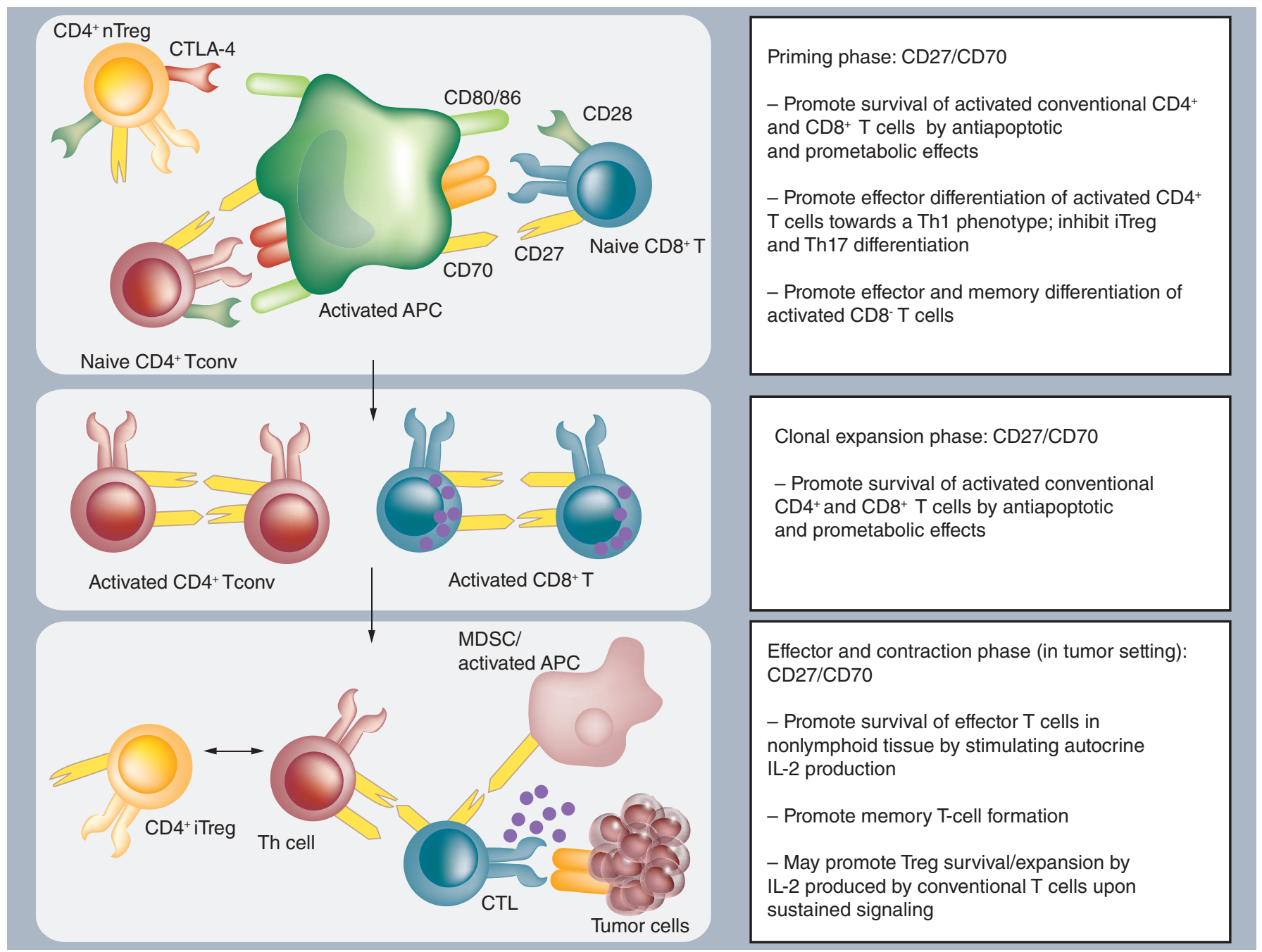

Figure 2. Mechanisms of action of the CD27/CD70 co-stimulatory system in regulation of the T-cell response. Depicted are the sequential phases in the T-cell response: priming (top panel), clonal expansion (middle panel) and effector phase (bottom panel). During priming, peptide/MHC complex on the APC may be recognized by the TCR. The nTreg inhibits the response of CD4 ${ }^{+}$Tconv and $C D 8^{+} \mathrm{T}$ cells to self-peptides, in part by sequestering CD80/CD86 by means of its cell surface CTLA-4. Alternatively, when nonself peptides are presented, CD80/CD86 is upregulated and CD70 is newly induced. These ligands trigger, respectively, CD28 and CD27 on the CD4+ Tconv cell and the CD8 ${ }^{+} \mathrm{T}$ cell. Together with the TCR signal, this induces clonal expansion and effector differentiation, in concert with cytokines and other signals delivered by the APC. During clonal expansion and after arrival of the effector T cells in nonlymphoid tissue, CD27/CD70 interactions continue to contribute to the T-cell response in the indicated fashion. It is not known which signals CD27 can deliver into Tregs.

APC: Antigen-presenting cell; CTL: Cytotoxic T lymphocyte; iTreg: Induced regulatory T cell; MDSC: Myeloid-derived suppressor cell; nTreg: Natural regulatory T cell; Tconv: Conventional CD4 ${ }^{+} \mathrm{T}$ cell.

and $\mathrm{CD}^{+} \mathrm{T}$ cell differentiation in various ways, as outlined here.

When a $\mathrm{CD}^{+} \mathrm{T}$ cell recognizes peptide/MHC class II complex on the DC, it becomes activated and upregulates CD40 ligand. The resulting CD40 signaling into the DC induces or upregulates CD70 expression. Newly synthesized CD70 protein travels to late endosomes that also contain MHC class II. Next, CD70 and MHC class II travel to the immunological synapse where they respectively stimulate CD27 and the TCR on the $\mathrm{CD}^{+} \mathrm{T}$ cell $[50,51]$. CD27 co-stimulation promotes Th1-type effector differentiation of the $\mathrm{CD}^{+} \mathrm{T}$ cell, as found consistently in human and mouse [40,43,52,53] (Figure 2). In human, CD27 co-stimulation was shown to promote $\mathrm{Th} 1$ differentiation by facilitating expression of the master transcription factor T-bet that is induced by IL-12 [43,52]. However, in the mouse, CD27 co-stimulation could promote Th1 differentiation by an IL-12-independent mechanism [53]. CD27 co-stimulation also inhibits Th17 differentiation in the mouse. It silenced expression of IL17 and CCR6 at the transcriptional level, after induction of the master transcription factor ROR $\gamma$ by TGF $\beta$ and IL-6 [54]. CD27/CD70 interaction may likewise inhibit human Th17 cell function [55]. 
$\mathrm{CD}^{+}{ }^{+} \mathrm{T}$-cell help is important for effector and memory differentiation of recently activated $\mathrm{CD} 8^{+} \mathrm{T}$ cells. This help is largely delivered via the DC [13]. Herein, the $\mathrm{CD}^{+} \mathrm{T}$ cell instructs the $\mathrm{DC}$ to adopt the optimal configuration to promote the differentiation of $\mathrm{CD}^{+} \mathrm{T}$ cells into functional CTLs as well as optimal memory cells (Figure 2). Immunological memory not only relies on the number of memory cells formed, but also on the intrinsic capacity of each memory cell to undergo secondary expansion. T cells acquire memory capacity by a differentiation process that appears to be initiated during priming [13]. DCs that are activated by PRR signals can instruct CTL effector differentiation in absence of $\mathrm{CD}^{+} \mathrm{T}$ cell help, but CTL memory differentiation always requires $\mathrm{CD} 4^{+} \mathrm{T}$ cell help [13]. This is the reason that therapeutic vaccines are formulated to contain both MHC class I and class II peptides [56]. When the DC expresses CD70, this supports CTL priming [20,34] and memory differentiation directly by $\mathrm{CD} 27$ signaling into $\mathrm{CD}^{+} \mathrm{T}$ cells [57], but also indirectly by $\mathrm{CD} 27$ signaling into $\mathrm{CD}^{+} \mathrm{T}$ cells [40]. Strong CD27 signaling into the $\mathrm{CD} 8{ }^{+} \mathrm{T}$ cell by, for example, CD70 transgenic DCs or recombinant CD70 bypasses the requirement of $\mathrm{CD}^{+}{ }^{+} \mathrm{T}$-cell help for CTL effector and memory responses [35,58].

Collective findings indicate that CD27 co-stimulation is very important for memory differentiation of $\mathrm{CD}^{+} \mathrm{T}$ cells. However, it is not absolutely required for the development of CTL effector capability [14,34-36,46]. Nevertheless, CD27 co-stimulation can promote IFN $\gamma$ production $[14,49,59-60]$ and cytotoxicity $[49,60]$ on a per cell basis. CD27 co-stimulation is not required for the exertion of CTL effector function, as indicated by the fact that terminally differentiated effector cells loose CD27 expression and CD70 is often absent from CTL target cells.

Memory T cells may not require CD27/CD70 costimulation during their secondary expansion. Upon protein immunization, deliberate CD27 stimulation with recombinant CD70 during T-cell priming strongly promoted the memory $\mathrm{CD}^{+}{ }^{+} \mathrm{T}$-cell response to immunization, while it had no effect when added during the recall [35]. Similar data were obtained in an influenza virus infection model [44]. However, CD27 signaling was important during the alloreactive memory response in a transplantation model [61] and during the secondary expansion of cytomegalovirus (CMV)-reactive T cells [62]. Such discrepancies are most likely related to the biology of virus infection and the related tissue specific contributions of CD27/CD70 co-stimulation. In intranasal influenza virus infection, co-stimulation by 4-1BB and OX40 likewise endowed CD8 ${ }^{+} \mathrm{T}$ cells with the capacity for secondary expansion during priming and was not needed during secondary expansion [63].
The collective data argue that CD27 co-stimulation is not absolutely required for effector differentiation of $\mathrm{CD}^{+}$or $\mathrm{CD}^{+} \mathrm{T}$ cells. However, in newly activated $\mathrm{CD} 4^{+} \mathrm{T}$ cells, CD27 co-stimulation can promote Th1 differentiation and can inhibit Th17 differentiation. In $\mathrm{CD}^{+} \mathrm{T}$ cells, CD27 signaling can promote CTL differentiation and is important for memory differentiation (Figure 2). The molecular basis of the latter effect remains to be revealed. Thus, CD70 induction on DCs is very important for the development of optimal primary and memory CTL responses. This underlines the potential of applying CD27 agonism in therapeutic vaccination against cancer and infectious disease.

\section{Contribution of CD27/CD70 to effector T-cell survival \& memory T-cell formation}

CD27/CD70 co-stimulation is also important after priming when effector $\mathrm{T}$ cells have relocated to nonlymphoid tissue effector sites [24]. In a model of intranasal influenza virus infection, accumulation of virusspecific $\mathrm{CD}^{+}$and $\mathrm{CD}^{+}$effector $\mathrm{T}$ cells in the lung was severely affected by CD27 deficiency [34,37-39,42,63]. Others also found that CD27 co-stimulation is required later in the immune response [44]. CD27 costimulation persists in the effector phase, because foreign antigen induces expression of CD70 on B cells and DCs in infected nonlymphoid tissue. The resulting CD27/CD70 interactions drive the survival of effector $\mathrm{T}$ cells [63]. For $\mathrm{CD}^{+} \mathrm{T}$ cells, the underlying mechanism has been identified: CD27 co-stimulation drives expression of the $\mathrm{Il}-2$ gene and enables effector T-cell survival in nonlymphoid tissue by autocrine IL-2 signaling [38]. During T-cell priming in the lymph nodes, autocrine IL-2 signaling does not contribute to T-cell survival and clonal expansion $[38,42]$. Thus the mechanisms by which CD27/CD70 co-stimulation promote the survival of activated $\mathrm{T}$ cells in lymphoid versus nonlymphoid tissue are distinct (Figure 2).

In the mouse, CD27 has been shown to act together with its close family members $4-1 \mathrm{BB}$ and OX40 in promoting effector $\mathrm{CD}^{+}$and $\mathrm{CD}^{+}{ }^{+}$T-cell survival in nonlymphoid tissue and in the formation of $\mathrm{CD}^{+}$ T cell memory cells [63]. The collective findings suggest that $4-1 \mathrm{BB}$ and to a lesser extent OX40 promote the survival of effector $\mathrm{CD}^{+} \mathrm{T}$ cells and their recruitment into the memory pool by the same mechanism as CD27. Their complementarity apparently lies in the different timing of receptor and/or ligand expression. CD27 and 4-1BB act sequentially on same CD8 ${ }^{+}$ $\mathrm{T}$ cells, as proven by the fact that $4-1 \mathrm{BB}$ is a CD27 target gene [39]. All infection or immunization models used support that CD27/CD70 co-stimulation promotes the formation of $\mathrm{CD}^{+}$memory $\mathrm{T}$ cells $[14,44,49,64]$. It does so by increasing the number of effector $T$ cells, but 
also by counteracting T-cell death during the contraction phase, as clearly demonstrated in CD70 transgenic mice $[49,58]$.

Co-stimulation by CD27 at the effector site, but also by 4-1BB and OX40 is driven by the local presence of their ligands on activated B cells and DCs [63]. This keeps the effector $\mathrm{CD}^{+}{ }^{+}$and $\mathrm{CD} 8{ }^{+}$effector $\mathrm{T}$ cells alive, as shown for $\mathrm{CD}^{+} \mathrm{T}$ cells by autocrine IL-2 signaling. Presumably, when antigen wanes, expression of the TNF ligands disappears and thereby effector $\mathrm{T}$ cells lose their survival support (Figure 2). The cells that continue to profit from CD27, 4-1BB and/or OX40 co-stimulation are recruited into the memory pool. It has been shown in many models that CD27/CD70 co-stimulation promotes the memory $\mathrm{CD} 8^{+} \mathrm{T}$-cell response, such as in infections with influenza virus [37,41,63], or CMV [62] and in immunization with proteins [35,40] or cells [57]. It appears to do so by incrementing the size of the CTL memory pool, as well as by promoting the capacity of secondary expansion in newly primed CTLs.

The CD27/CD70 pathway \& regulatory T cells Apart from affecting conventional T-cell responses, CD27-targeted immunotherapy may also affect Treg responses. Tregs expand particularly in chronic immune activation and cancer. Their function is to prevent autoimmunity at steady-state and when the T-cell response is (over)activated [18]. Consequently, Tregs can impede both priming and effector function of CTLs and indeed, intratumoral Tregs are associated with poor prognosis of cancer patients [5,18]. The effect of CD27/CD70 co-stimulation on Treg biology is complex. Tregs are discriminated into nTregs, that develop in the thymus and have an autoreactive TCR repertoire and induced (i)Tregs, that develop from conventional $\mathrm{CD}^{+} \mathrm{T}$ cells during the immune response. CD27/CD70 co-stimulation promotes the thymic development of nTregs, as has been shown in the mouse. CD70 on mTECs and thymic DCs interacts with CD27 on newly formed nTregs to promote their escape from negative selection by antiapoptotic signaling [26]. The same may be the case in human, where CD70 is also constitutively expressed on mTECs [27].

Foxp $3^{+}$Tregs may or may not express CD27 [31,65,66]. Human $\mathrm{CD} 27^{+}$Treg proved more potent suppressors than CD27- Tregs, but suppression was independent of CD27 signaling [31]. When considering the impact of CD27/CD70 on Tregs, it is important to discriminate between transient and constitutive engagement of this co-stimulatory system. Claus et al. [66] reported that ongoing CD27/CD70 interactions during tumor development promoted Treg expansion in tumor-bearing mice and thereby frustrated the CTL response against the tumor. Treg expansion depended on IL-2 produced by conventional $\mathrm{T}$ cells. Thus, when CD27/CD70 costimulation promotes effector $\mathrm{CD}^{+}$and $\mathrm{CD}^{+}{ }^{+} \mathrm{T}$ cell survival by inducing autocrine IL-2 signaling, Tregs can profit from this (Figure 2).

Under conditions of transient CD27/CD70 costimulation, such as in acute infection or immunization, CTL responsiveness appeared dominant over potential Treg expansion, since effective, antiviral or antitumor responses were raised. In cancer immunotherapy, CD27 will likewise be transiently engaged. It should be considered how such an intervention will affect a pre-existing intratumoral Treg population. CD27 stimulation on human Tregs has been found to diminish their suppressive function and convert them into Th1 cells [52]. In addition, CD27 co-stimulation is critical for new $\mathrm{T}$ cell priming. Therefore, CD27 agonism is expected to be a favorable intervention even when tumors contain Tregs.

\section{Consequences of constitutive CD27/CD70 engagement}

In chronic immune activation, such as in autoimmunity or HIV infection, CD70 is persistently expressed, at least at the surface of $T$ cells [23]. Mice with constitutive transgenic expression of CD70 on DCs or B cells highlight that $\mathrm{CD} 27$ co-stimulation alters the threshold for $\mathrm{CD}^{+}$and $\mathrm{CD}^{+}$T-cell priming. When such transgenic mice are housed in animal facilities that are not specific pathogen-free, constitutive CD27/CD70 co-stimulation drives the generation of $\mathrm{CD}^{+}$and $\mathrm{CD}^{+}$effector $\mathrm{T}$ cells in the absence of deliberate immunization. This response is TCR-driven, by environmental and autoantigens. Upon ageing, the mice develop combined $\mathrm{T}$ and $\mathrm{B}$ cell deficiency and die due to opportunistic infections [67]. This happens because all naive $\mathrm{T}$ cells turn into effector $\mathrm{T}$ cells and effector T-cell derived IFN $\gamma$ blocks new B-cell development in the bone marrow [59]. CD70 transgenic mice do not develop autoimmunity. The arising activated $T$ cells die as a result of CD95-mediated apoptosis [64,68]. CD70 transgenic mice that are CD95-decifient die early, due to massive tissue infiltration by effector $\mathrm{T}$ cells [68].

Clearly, the consequences of transient versus constitutive engagement of CD27 are distinct, as was also demonstrated in mice with acute versus chronic infection with lymphocytic choriomeningitis virus. Herein, constitutive engagement of CD27/CD70 was shown to promote T-cell exhaustion, in other words, loss of effector functions [69], as has also been found in human B cell lymphoma [70]. Thus the CD27/CD70 co-stimulatory system has built-in negative control mechanisms, i.e. T-cell exhaustion and CD95-mediated apoptosis that attenuate the generation of effector $\mathrm{T}$ cells when antigenic stimulation persists. 


\section{Impact of CD27/CD70 co-stimulation on other immune cell types}

In both human and mouse, CD27 is absent from naive $\mathrm{B}$ cells and induced upon their activation [24,25]. CD27/CD70 co-stimulation promotes the B-cell response by supporting $\mathrm{B}$-cell expansion in the germinal center, in a B-cell intrinsic way and by promoting $\mathrm{CD} 4{ }^{+} \mathrm{T}$-cell help. In this way, CD27/CD70 co-stimulation promotes the generation of effector and memory $\mathrm{B}$ cells, but does not drive the B-cell differentiation program as such $[24,25,71]$.

Human NK cells express CD27 depending on their stage in effector differentiation [72]. The CD27/CD70 pathway does not directly influence NK effector function, but can increase IFN $\gamma$ levels in NK cells, which in turn enhances their cytotoxic capability [73]. Agonistic $\alpha \mathrm{CD} 27 \mathrm{mAb}$ slowed growth of CD27- tumors in T-cell deficient mice, indicating a contribution of NK cells [74]. Furthermore, CD70 expression on MHC class I-deficient lymphoma cells promoted their rejection by NK cells and induced T-cell memory [75]. Thus, the CD27/CD70 pathway promotes B-cell and NK cell activity, which can contribute to tumor control.

\section{Evaluating the effect of deliberate CD27 co- stimulation in cancer}

In mouse models, the efficacy of CD27 agonism to promote immunity to cancer has been evaluated by various strategies. In mice with transgenic expression of CD70 on B cells or DCs, antitumor immunity was improved against EL-4 lymphoma and B16 melanoma tumors bearing foreign antigen, but also against the parental tumors [47,49]. Peptide-loaded CD70 transgenic DCs were also effective in a vaccination setting [58]. Treatment of mice with agonistic $\mathrm{mAb}$ directed at mouse CD27 likewise improved antitumor immunity against T- and B-cell lymphoma and B16 melanoma [60,74,76].

The action of agonistic $\mathrm{mAb}$ directed against human CD27 was shown by Vitale et al. [77] and He et al. [78], who reported the immunomodulatory effects of the fully human $1 \mathrm{~F} 5 \mathrm{mAb}$ that is now in Phase I clinical testing in B-cell malignancies, melanoma and renal cell carcinoma as CDX-1127 (varlilumab). As expected from triggering a co-stimulatory receptor, this $\mathrm{mAb}$ promotes the response of human $\mathrm{T}$ cells that are activated by TCR signaling, but does not produce a response in absence of TCR signaling [77,78]. This is essential, since general T-cell activation in the absence of TCR specificity is lethal, as painfully illustrated by the unsuccessful trial with the CD28 superagonist $\mathrm{mAb}$ TGN1412 [79]. The 1F5 mAb is an IgG1 that can bind to Fc receptors. In fact, Fc receptor binding is required for its agonistic activity [77]. There is a risk that the $\mathrm{mAb}$ thereby depletes CD27-bearing cells due to phagocytosis or NK-mediated killing. This was tested in cynomolgous and rhesus macaques whose CD27 is recognized with similar affinity by $1 \mathrm{~F} 5 \mathrm{mAb}$ as human CD27 [77]. In these primates, CD27 expression on $\mathrm{T}$ and $\mathrm{B}$ cells was very comparable to human, but it was more abundant on NK cells. 1F5 mAb treatment at 1 , 3 or $10 \mathrm{mg} / \mathrm{kg}$ induced a transient increase in blood $\mathrm{CD}^{+} \mathrm{T}$ cells and a decrease in $\mathrm{CD} 8^{+} \mathrm{T}$ cells. NK cell numbers transiently dropped by $60-90 \%$. Treg numbers decreased by $25-50 \%$, but only at higher doses of 1F5. This effect was still present at the end of monitoring (day 29). 1F5 mAb treatment did not induce toxicity as determined by temperature, body weight and cytokine levels in blood. The efficacy of 1F5 mAb treatment to induce T-cell dependent immunity was tested in mice transgenic for the human CD27 gene locus, including its regulatory elements [78]. In these mice, human CD27 has the same expression pattern as in human. Stimulation of human or mouse CD27 with 1F5 $\mathrm{mAb}$ or antimouse $\mathrm{CD} 27$ agonist $\mathrm{mAb}$ enhanced the CD8 ${ }^{+} \mathrm{T}$-cell response to protein immunization in a comparable fashion. $1 \mathrm{~F} 5 \mathrm{mAb}$ treatment also produced $\mathrm{CD} 4-^{+}$and $\mathrm{CD} 8{ }^{+}$T-cell dependent therapeutic effects against s.c. implanted CT26 colon carcinoma and against $\mathrm{T}$ lymphoma cells bearing foreign antigen, but not to the parental lymphoma cells [78]. The treated mice were resistant to subsequent tumor challenge, showing that $\mathrm{CD} 8{ }^{+}$effector $\mathrm{T}$ memory had been established. Progress of clinical trials with varlilumab can be followed on internet.

Note: Mechanistic knowledge of the CD27/CD70 co-stimulatory system is for a large part based on studies in mice. Its importance for human immunity has however been confirmed by the discovery of patients with genetic CD27 deficiency. These patients are predisposed for immunodeficiency with potentially fatal Epstein-Barr Virus-driven hemophagocytosis and lymphoma development, memory B-cell deficiency and T-cell dysfunction $[80,81]$. These symptoms fit with an important role of CD27/CD70 co-stimulation in the control of (viral) infection and are remarkably consistent with the mechanism of action deduced from mouse studies.

\section{Conclusion \& future perspective}

On the basis of the experimental evidence reviewed here, it can be stated that the CD27/CD70 co-stimulatory system is geared to promote T, B and NK cell responses and particularly the CTL response, which inspires its targeting in cancer immunotherapy. The focus of cancer immunotherapy should be on immunogenic tumors that can be recognized by $\mathrm{CD} 8^{+} \mathrm{T}$ cells. Identification of tumor antigens by deep sequencing, combined with accurate diagnosis of tumor-specific 
T cells as carried out by Kvistborg et al. [7] will help in the identification of eligible cancer types. Furthermore, in the future, immunotherapy will hopefully be applied in earlier stages of cancer development, increasing its chance of success.

Peripheral tolerance to the tumor, that is, the lack of DC activation is a key limitation in raising CTL responses to cancer that $\mathrm{CD} 27$ agonism can help to overcome. Deliberate CD27 co-stimulation will promote the activation of $\mathrm{T}$ cells, their clonal expansion and effector differentiation, the survival of effector
$\mathrm{T}$ cells at the tumor site and T-cell memory. Deliberate CD27 co-stimulation can engage NK cells and B cells to facilitate tumor cell killing. It remains to be seen whether deliberate CD27 co-stimulation can help to alleviate immunosuppression at the tumor site, but current data suggest that this is not a key feature of CD27 co-stimulation. Therefore, CD27 agonism is expected to be most successful when used in combination with other forms of therapy that specifically address this bottleneck. Indeed, current data from a Phase I monotherapy trial with CDX-1127 in hemato-

\section{Executive summary}

\section{The promise of cancer immunotherapy}

- Apart from blocking T-cell coinhibitory receptors, deliberate stimulation of T-cell co-stimulatory receptors, including CD27, is expected to increase the success of cancer immunotherapy with immunomodulatory monoclonal antibodies.

\section{Introduction CD27 \& CD70}

- CD27 is a member of the TNF receptor family that is specifically expressed by cells of the lymphoid lineage, including naive $\mathrm{CD}^{+}$and $\mathrm{CD} 8^{+} \mathrm{T}$ cells.

- Expression of CD70, the CD27 ligand, is tightly controlled. It is present on dendritic cells (DCs), B cells, T cells and NK cells, but only after their activation.

\section{Contribution of CD27/CD70 to T-cell activation \& clonal expansion}

- During priming, CD27/CD70 co-stimulation is complementary to CD28 co-stimulation. It promotes clonal expansion of antigen-specific $\mathrm{CD} 4^{+}$and $\mathrm{CD} 8^{+} \mathrm{T}$ cells by IL-2-independent survival signaling.

- CD27 signaling counteracts apoptosis of activated T cells throughout successive divisions and has prometabolic effects.

- As a result, CD27/CD70 co-stimulation promotes the generation of effector T cells in lymphoid organs and broadens the responder T-cell repertoire.

Contribution of CD27-CD70 to effector \& memory T-cell differentiation

- $C D 27 / C D 70$ co-stimulation is not required for $\mathrm{CD}^{+}$or $\mathrm{CD} 8{ }^{+}$effector differentiation, but evidently promotes T helper-1 differentiation of $\mathrm{CD}^{+}{ }^{+} \mathrm{T}$ cells and supports effector differentiation of cytotoxic T-lymphocyte (CTL).

- The CD27/CD70 pathway plays a very important role in memory differentiation of CD8+ ${ }^{+}$cells.

Contribution of CD27/CD70 to effector T-cell survival \& T-cell memory formation

- CD27/CD70 co-stimulation promotes the survival of effector T cells at tissue sites outside of the lymphoid organs via autocrine IL-2 signaling. It also promotes the formation of memory CD $8^{+} \mathrm{T}$ cells.

The CD27/CD70 pathway \& regulatory T cells

- CD27/CD70 co-stimulation counteracts conversion of conventional CD4+ $T$ cells into iTregs, but can also promote thymic differentiation of nTregs and, upon sustained signaling, promote clonal expansion of Tregs via IL-2 made by Tconv.

Consequences of constitutive CD27/CD70 engagement

- Transient CD27/CD70 co-stimulation promotes the T-cell response, but constitutive CD27/CD70 co-stimulation as occurs in chronic viral infection, leads to T-cell exhaustion and death.

Impact of CD27/CD70 co-stimulation on other immune cell types

- Transient CD27/CD70 co-stimulation promotes the B- and NK-cell response, which can contribute to tumor control.

Evaluating the effect of deliberate CD27 co-stimulation in cancer

- Deliberate, transient CD27/CD70 co-stimulation by agonistic antibody to CD27, soluble CD70 or CD70-bearing DCs promotes the CTL effector response and CTL memory to cancer.

Conclusion \& future perspective

- CD27 agonism in cancer immunotherapy will help to overcome T-cell tolerance, that is, the lack of DC activation. There is no evidence that it can overrule tumor-associated immune suppression.

- CD27 agonism is expected to act additively or synergistically with PD-1/L-1 blocking and may replace CTLA-4 blocking in combined treatment.

- CD27 agonism is expected to improve results of therapeutic vaccination and radiotherapy, alone or in combination with PD-1/L-1 blocking. 
logic malignancy indicate antitumor activity, including one complete remission, but there is ample room for improvement [82].

Combining agonistic mAb CD27 with blocking $\mathrm{mAb}$ to CTLA- 4 or PD-1/-L1 is expected to have an additive or even synergistic effect on the antitumor response. CD27 agonism and CTLA- 4 blocking are expected to have a combined effect on the $\mathrm{T}$ cell response, since $\alpha \mathrm{CTLA}-4$ promotes $\mathrm{CD} 28$ costimulation [19]. CD27 agonism and PD-1/-L1 blocking are expected to have a combined effect on the T-cell response, because the latter will alleviate suppression of CTLs in the tumor microenvironment. Ideally, an agonist $\mathrm{mAb}$ is used that has CD27 stimulatory activity in absence of $\mathrm{Fc}$ receptor binding, thus decreasing the risk of immune cell depletion.

$\mathrm{CD} 27$ agonist mAb may also be applied in therapeutic vaccination against cancer. The success of this approach is thus far limited, but may be increased by combination of CD27 agonism with PD-1/-L1 blocking. Along the same lines, CD27 agonism can be combined with chemo- or radiotherapy that promote tumor-cell death and hence enhance the presentation of tumor antigens. Apart from using mAb, CD27 agonism can also be achieved by using soluble CD70 or it can be incorporated in DC therapy. In the TriMix therapy that is currently in clinical testing for melanoma treatment, monocyte-derived DCs are transfected with mRNA encoding for tumor antigens, CD70, CD40L and constitutive active TLR4 [52]. In vaccination approaches, tumor-specific $T$ cells are activated, but not $\mathrm{T}$ cells with other specificities, which is expected to limit the therapy-related adverse effects.

$\mathrm{CD} 27$ agonism with $\mathrm{mAb}$ or CD70-bearing DCs as stand-alone treatment is well-tolerated, with only minor side effects such as fatigue and fever [82,83]. In contrast, with $\alpha$ CTLA4 treatment adverse autoimmune effects are seen in about $60 \%$ of patients [1] and adverse effects were more severe when ipilimumab and nivolumab were combined [2]. We suggest that CD27 agonism may be a good alternative for CTLA- 4 blocking and may be optimally combined with PD-1/

\section{References}

Papers of special note have been highlighted as:

- of interest; $\bullet \bullet$ of considerable interest

1 Hodi FS, O'Day SJ, McDermott DF et al. Improved survival with ipilimumab in patients with metastatic melanoma. N. Engl. J. Med. 363(8), 711-723 (2010).

2 Wolchok JD, Kluger H, Callahan MK et al. Nivolumab plus ipilimumab in advanced melanoma. N. Engl. J. Med. 369(2), 122-133 (2013).
PD-L1 blocking. In therapy with CD27 agonism, cancers that express CD27 deserve special attention. Thus far, however, CD27 agonism does not seem to affect survival or proliferation of such cells, but rather to promote their T-cell mediated killing. Co-stimulation with agonistic CD27 mAb may also be applied during in vitro expansion of tumor-infiltrating $\mathrm{T}$ cells intended for adoptive cell therapy, in order to maintain tumor-specific T-cell clones. A CD27-positive phenotype of tumor-infiltrating $\mathrm{T}$ cells favors longterm in vivo persistence of the cells, as well as clinical outcome in cancer therapy, as expected from the data on CD27/CD70 function [84].

As widely acknowledged, there is great potential for the use of immunomodulating $\mathrm{mAbs}$ in cancer therapy. Therapy at earlier stages of disease, using $\mathrm{mAb}$ combinations alone or together with therapeutic vaccination, is expected to increase disease control and potentially curation in a great variety of cancers.

\section{Financial \& competing interests disclosure}

This review is extracted by the authors from a Masters student thesis written by $K$ van de Ven, as part of his graduation requirements at the University of Amsterdam. Work in the laboratory of J Borst on CD27/CD70 co-stimulation is supported by grants from The Dutch Cancer Society and The Netherlands Organization for Scientific Research. J Borst has a scientific collaboration with the biotech company BioNovion. She is listed as co-inventor on a patent application titled 'Agonistic antibody to CD27.' The authors have no other relevant affiliations or financial involvement with any organization or entity with a financial interest in or financial conflict with the subject matter or materials discussed in the manuscript apart from those disclosed.

No writing assistance was utilized in the production of this manuscript.

\section{Open Access}

This work is licensed under the Creative Commons Attribution-NonCommercial 3.0 Unported License. To view a copy of this license, visit http://creativecommons.org/licenses/bync-nd/3.0/

3 Topalian SL, Hodi FS, Brahmer JR et al. Safety, activity, and immune correlates of anti-PD-1 antibody in cancer. N. Engl. J. Med. 366(26), 2443-2454 (2012).

4 Couzin-Frankel J. Breakthrough of the year 2013. Cancer immunotherapy. Science 342(6165), 1432-1433 (2013).

5 Gajewski TF, Schreiber H, Fu Y-X. Innate and adaptive immune cells in the tumor microenvironment. Nat. Immunol. 14(10), 1014-1022 (2013).

6 Alexandrov LB, Nik-Zainal S, Wedge DC et al. Signatures of mutational processes in human cancer. Nature 500 (7463), 415-421 (2013). 
7 Kvistborg P, Philips D, Kelderman S et al. Anti-CTLA-4 therapy broadens the melanoma-reactive $\mathrm{CD} 8{ }^{+} \mathrm{T}$ cell response. Sci. Transl. Med. 6(254), 254ra128-254ra128 (2014).

8 Tumeh PC, Harview CL, Yearley JH et al. PD-1 blockade induces responses by inhibiting adaptive immune resistance. Nature 515(7528), 568-571 (2014).

9 Herbst RS, Soria J-C, Kowanetz M et al. Predictive correlates of response to the anti-PD-L1 antibody MPDL3280A in cancer patients. Nature 515(7528), 563-567 (2014).

10 Aranda F, Vacchelli E, Eggermont A et al. Trial watch: immunostimulatory monoclonal antibodies in cancer therapy. Oncoimmunology 3(1), e27297 (2014).

11 Acuto O, Michel F. CD28-mediated co-stimulation: a quantitative support for TCR signalling. Nat. Rev. Immunol. 3(12), 939-951 (2003).

12 Tan JKH, O’Neill HC. Maturation requirements for dendritic cells in $\mathrm{T}$ cell stimulation leading to tolerance versus immunity. J. Leukoc. Biol. 78(2), 319-324 (2005).

13 Bevan MJ. Helping the CD8(+) T-cell response. Nat. Rev. Immunol. 4(8), 595-602 (2004).

14 Taraban VY, Rowley TF, Al-Shamkhani a. Cutting edge: a critical role for CD70 in CD8 T cell priming by CD40licensed APCs. J. Immunol. 173(11), 6542-6546 (2004).

15 Tesselaar K, Xiao Y, Arens R et al. Expression of the murine CD27 ligand CD70 in vitro and in vivo. J. Immunol. 170(1), 33-40 (2003).

16 Bullock TNJ, Yagita H. Induction of CD70 on dendritic cells through CD 40 or TLR stimulation contributes to the development of $\mathrm{CD}^{+} \mathrm{T}$ cell responses in the absence of $\mathrm{CD} 4^{+}$ T cells. J. Immunol. 174(2), 710-717 (2005).

17 Sanchez PJ, McWilliams JA, Haluszczak C, Yagita H, Kedl RM. Combined TLR/CD40 stimulation mediates potent cellular immunity by regulating dendritic cell expression of CD70 in vivo. J. Immunol. 178(3), 1564-1572 (2007).

18 Zou W. Regulatory T cells, tumour immunity and immunotherapy. Nat. Rev. Immunol. 6(4), 295-307 (2006).

19 Onishi Y, Fehervari Z, Yamaguchi T, Sakaguchi S. Foxp3 ${ }^{+}$ natural regulatory $\mathrm{T}$ cells preferentially form aggregates on dendritic cells in vitro and actively inhibit their maturation. Proc. Natl. Acad. Sci. 105(29), 10113-10118 (2008).

20 Bodmer J-L, Schneider P, Tschopp J. The molecular architecture of the TNF superfamily. Trends Biochem. Sci. 27(1), 19-26 (2002).

21 Aggarwal BB. Signalling pathways of the TNF superfamily: a double-edged sword. Nat. Rev. Immunol. 3(9), 745-756 (2003).

22 Watts TH. TNF/TNFR family members in costimulation of T cell responses. Annu. Rev. Immunol. 23, 23-68 (2005).

23 Nolte MA, Van Olffen RW, Van Gisbergen KPJM, Van Lier RAW. Timing and tuning of CD27-CD70 interactions: the impact of signal strength in setting the balance between adaptive responses and immunopathology. Immunol. Rev. 229(1), 216-231 (2009).

24 Borst J, Hendriks J, Xiao Y. CD27 and CD70 in T cell and B cell activation. Curr. Opin. Immunol. 17(3), 275-281 (2005).
25 Lens SM, Tesselaar K, van Oers MH, van Lier RA. Control of lymphocyte function through CD27-CD70 interactions. Semin. Immunol. 10 (6), 491-499 (1998).

26 Coquet JM, Ribot JC, B bała N et al. Epithelial and dendritic cells in the thymic medulla promote $\mathrm{CD} 4^{+} \mathrm{Foxp}^{+}$regulatory $\mathrm{T}$ cell development via the CD27-CD70 pathway. J. Exp. Med. 210(4), 715-728 (2013).

27 Hintzen RQ, Lens SMA, Koopman G, Pals ST, Spits H, van Lier RAW. CD70 represents the human ligand for CD27. Int. Immunol. 6(3), 477-480 (1994).

28 Laouar A, Haridas V, Vargas D et al. CD70+ antigenpresenting cells control the proliferation and differentiation of $\mathrm{T}$ cells in the intestinal mucosa. Nat. Immunol. 6(7), 698-706 (2005).

29 Iwamoto S, Iwai S, Tsujiyama K et al. TNF-alpha drives human $\mathrm{CD} 14^{+}$monocytes to differentiate into CD70+ dendritic cells evoking Th1 and Th17 responses. J. Immunol. 179(3), 1449-1457 (2007).

30 Krause P, Bruckner M, Uermösi C, Singer E, Groettrup M, Legler DF. Prostaglandin E(2) enhances T-cell proliferation by inducing the costimulatory molecules OX40L, CD70, and 4-1BBL on dendritic cells. Blood 113(11), 2451-2460 (2009).

31 Koenen HJPM, Fasse E, Joosten I. CD27/CFSE-based ex vivo selection of highly suppressive alloantigen-specific human regulatory T cells. J. Immunol. 174(12), 7573-7583 (2005).

32 Van Lier RA, Borst J, Vroom TM et al. Tissue distribution and biochemical and functional properties of $\mathrm{Tp} 55$ (CD27), a novel $\mathrm{T}$ cell differentiation antigen. J. Immunol. 139(5), 1589-1596 (1987).

33 Goodwin RG, Alderson MR, Smith CA et al. Molecular and biological characterization of a ligand for $\mathrm{CD} 27$ defines a new family of cytokines with homology to tumor necrosis factor. Cell 73(3), 447-456 (1993).

34 Hendriks J, Gravestein LA, Tesselaar K, van Lier RA, Schumacher TN, Borst J. CD27 is required for generation and long-term maintenance of $\mathrm{T}$ cell immunity. Nat. Immunol. 1(5), 433-440 (2000).

35 Rowley TF, Al-Shamkhani A. Stimulation by soluble CD70 promotes strong primary and secondary $\mathrm{CD} 8^{+}$cytotoxic T cell responses in vivo. J. Immunol. 172(10), 6039-6046 (2004).

36 Schildknecht A, Miescher I, Yagita H, van den Broek M. Priming of $\mathrm{CD}^{+} \mathrm{T}$ cell responses by pathogens typically depends on CD70-mediated interactions with dendritic cells. Eur. J. Immunol. 37(3), 716-728 (2007).

37 Hendriks J, Xiao Y, Borst J. CD27 promotes survival of activated T cells and complements CD28 in generation and establishment of the effector T cell pool. J. Exp. Med. 198(9), 1369-1380 (2003).

38 Peperzak V, Xiao Y, Veraar EAM, Borst J. CD27 sustains survival of CTLs in virus-infected nonlymphoid tissue in mice by inducing autocrine IL-2 production. J. Clin. Invest. 120(1), 168-178 (2010).

39 Peperzak V, Veraar EAM, Xiao Y et al. CD8 ${ }^{+} \mathrm{T}$ cells produce the chemokine CXCL10 in response to CD27/CD70 
costimulation to promote generation of the $\mathrm{CD}^{+}$effector T cell pool. J. Immunol. 191(6), 3025-3036 (2013).

40 Xiao Y, Peperzak V, Keller AM, Borst J. CD27 instructs $\mathrm{CD} 4{ }^{+} \mathrm{T}$ cells to provide help for the memory $\mathrm{CD} 8^{+} \mathrm{T}$ cell response after protein immunization. J. Immunol. 181(2), 1071-1082 (2008).

41 Van Gisbergen KPJM, Klarenbeek PL, Kragten NAM et al. The costimulatory molecule CD27 maintains clonally diverse CD8 $\left(^{+}\right) \mathrm{T}$ cell responses of low antigen affinity to protect against viral variants. Immunity 35(1), 97-108 (2011).

- Data indicating that $\mathrm{CD} 27$ costimulation broadens the responder T-cell repertoire.

42 Peperzak V, Veraar EAM, Keller AM, Xiao Y, Borst J. The Pim kinase pathway contributes to survival signaling in primed $\mathrm{CD} 8^{+} \mathrm{T}$ cells upon $\mathrm{CD} 27$ costimulation. J. Immunol. 185(11), 6670-6678 (2010).

43 Van Oosterwijk MF, Juwana H, Arens R et al. CD27-CD70 interactions sensitise naive $\mathrm{CD}^{+}{ }^{+} \mathrm{T}$ cells for IL-12-induced Th1 cell development. Int. Immunol. 19(6), 713-718 (2007).

44 Dolfi D V, Boesteanu AC, Petrovas C, Xia D, Butz EA, Katsikis PD. Late signals from CD27 prevent Fas-dependent apoptosis of primary CD8 ${ }^{+}$T cells. J. Immunol. 180(5), 2912-2921 (2008).

45 Amaravadi R, Thompson CB. The survival kinases Akt and Pim as potential pharmacological targets. J. Clin. Invest. 115(10), 2618 (2005).

46 Carr JM, Carrasco MJ, Thaventhiran JED et al. CD27 mediates interleukin-2-independent clonal expansion of the $\mathrm{CD}^{+} \mathrm{T}$ cell without effector differentiation. Proc. Natl Acad. Sci. USA 103(51), 19454-19459 (2006).

47 Keller AM, Schildknecht A, Xiao Y, van den Broek M, Borst J. Expression of costimulatory ligand CD70 on steady-state dendritic cells breaks CD8 ${ }^{+} \mathrm{T}$ cell tolerance and permits effective immunity. Immunity 29(6), 934-946 (2008).

- Paper showing the potency of CD27/CD70 interactions to promote the success of therapeutic vaccination.

48 Probst HC, McCoy K, Okazaki T, Honjo T, van den Broek $\mathrm{M}$. Resting dendritic cells induce peripheral $\mathrm{CD} 8^{+} \mathrm{T}$ cell tolerance through PD-1 and CTLA-4. Nat. Immunol. 6(3), 280-286 (2005)

49 Arens R, Schepers K, Nolte MA et al. Tumor rejection induced by $\mathrm{CD} 70$-mediated quantitative and qualitative effects on effector $\mathrm{CD} 8^{+} \mathrm{T}$ cell formation. J. Exp. Med. 199(11), 1595-1605 (2004).

50 Zwart W, Peperzak V, de Vries E et al. The invariant chain transports TNF family member CD70 to MHC class II compartments in dendritic cells. J. Cell Sci. 123(21), 3817-3827 (2010).

51 Keller AM, Groothuis TA, Veraar EAM et al. Costimulatory ligand CD70 is delivered to the immunological synapse by shared intracellular trafficking with MHC class II molecules. Proc. Natl Acad. Sci. USA 104(14), 5989-5994 (2007).

52 Pen JJ, De Keersmaecker B, Maenhout SK et al. Modulation of regulatory $\mathrm{T}$ cell function by monocyte-derived dendritic cells matured through electroporation with mRNA encoding CD40 ligand, constitutively active TLR4, and CD70. J. Immunol. 191(4), 1976-1983 (2013).
- Data indicating that CD27 signaling counteracts conversion of conventional $\mathrm{CD} 4^{+} \mathrm{T}$ cells into Tregs.

53 Soares $\mathrm{H}$, Waechter $\mathrm{H}$, Glaichenhaus $\mathrm{N}$ et al. A subset of dendritic cells induces $\mathrm{CD}^{+}{ }^{+} \mathrm{T}$ cells to produce IFN-gamma by an IL-12-independent but CD70-dependent mechanism in vivo. J. Exp. Med. 204(5), 1095-1106 (2007).

54 Coquet JM, Middendorp S, van der Horst G et al. The CD27 and CD70 costimulatory pathway inhibits effector function of $\mathrm{T}$ helper 17 cells and attenuates associated autoimmunity. Immunity 38(1), 53-65 (2013).

55 Yang Z-Z, Novak AJ, Ziesmer SC, Witzig TE, Ansell SM. Malignant $B$ cells skew the balance of regulatory $T$ cells and TH17 cells in B-cell non-Hodgkin's lymphoma. Cancer Res. 69(13), 5522-5530 (2009).

56 Melief CJM, van der Burg SH. Immunotherapy of established (pre) malignant disease by synthetic long peptide vaccines. Nat. Rev. Cancer. 8(5), 351-360 (2008).

57 Feau S, Garcia Z, Arens R, Yagita H, Borst J, Schoenberger SP. The $\mathrm{CD}^{+}{ }^{+} \mathrm{T}$-cell help signal is transmitted from APC to CD8 ${ }^{+}$T-cells via CD27-CD70 interactions. Nat. Commun. 3(5), 948 (2012).

58 Keller AM, Xiao Y, Peperzak V, Naik SH, Borst J. Costimulatory ligand $\mathrm{CD} 70$ allows induction of $\mathrm{CD} 8^{+}$ T-cell immunity by immature dendritic cells in a vaccination setting. Blood 113(21), 5167-5175 (2009).

59 Arens R, Tesselaar K, Baars PA et al. Constitutive CD27/ $\mathrm{CD} 70$ interaction induces expansion of effector-type $\mathrm{T}$ cells and results in IFNgamma-mediated B cell depletion. Immunity 15(5), 801-812 (2001).

60 Roberts DJ, Franklin NA, Kingeter LM et al. Control of established melanoma by CD27 stimulation is associated with enhanced effector function and persistence, and reduced PD-1 expression of tumor infiltrating CD8 $\left(^{+}\right) \mathrm{T}$ cells. J. Immunother. 33(8), 769-779 (2010).

61 Yamada A, Salama AD, Sho M et al. CD70 signaling is critical for $\mathrm{CD} 28$-independent $\mathrm{CD} 8{ }^{+} \mathrm{T}$ cell-mediated alloimmune responses in vivo. J. Immunol. 174(3), 1357-1364 (2005).

62 Welten SPM, Redeker A, Franken KL et al. CD27-CD70 costimulation controls $\mathrm{T}$ cell immunity during acute and persistent cytomegalovirus infection. J. Virol. 87(12), 6851-6865 (2013).

63 Hendriks J, Xiao Y, Rossen JWA et al. During viral infection of the respiratory tract, CD27, 4-1BB, and OX40 collectively determine formation of $\mathrm{CD}^{+}$memory $\mathrm{T}$ cells and their capacity for secondary expansion. J. Immunol. 175(3), 1665-1676 (2005).

64 Wensveen FM, Unger P-PA, Kragten NAM et al. CD70driven costimulation induces survival or Fas-mediated apoptosis of T cells depending on antigenic load. J. Immunol. 188(9), 4256-4267 (2012).

65 Mack DG, Lanham AM, Palmer BE, Maier LA, Fontenot AP. CD27 expression on $\mathrm{CD}^{+} \mathrm{T}$ cells differentiates effector from regulatory $\mathrm{T}$ cell subsets in the lung. J. Immunol. 182(11), 7317-7324 (2009).

66 Claus C, Riether C, Schürch C, Matter MS, Hilmenyuk $\mathrm{T}$, Ochsenbein AF. CD27 signaling increases the frequency 
of regulatory $\mathrm{T}$ cells and promotes tumor growth. Cancer Res. 72(14), 3664-3676 (2012).

Tesselaar K, Arens R, van Schijndel GM, Baars PA, van der Valk MA, Borst J, van Oers MH, van Lier RA. Lethal T cell immunodeficiency induced by chronic costimulation via CD27-CD70 interactions. Nat. Immunol. 4(1), 49-54 (2003).

68 Arens R, Baars PA, Jak M et al. Cutting edge: CD95 maintains effector $\mathrm{T}$ cell homeostasis in chronic immune activation. J. Immunol. 174(10), 5915-5920 (2005). Penaloza-MacMaster P, Rasheed AU, Iyer SS, Yagita H, Blazar BR, Ahmed R. Opposing effects of CD70 costimulation during acute and chronic lymphocytic choriomeningitis virus infection of mice. J. Virol. 85(13), 6168-6174 (2011).

70 Yang Z-Z, Grote DM, Xiu B et al. TGF- $\beta$ upregulates CD70 expression and induces exhaustion of effector memory T cells in B-cell non-Hodgkin's lymphoma. Leukemia 28(9), 1872-1884 (2014).

71 Xiao Y, Hendriks J, Langerak P, Jacobs H, Borst J. CD27 is acquired by primed $\mathrm{B}$ cells at the centroblast stage and promotes germinal center formation. J. Immunol. 172(12), 7432-7441 (2004).

72 Vossen MTM, Matmati M, Hertoghs KML et al. CD27 defines phenotypically and functionally different human NK cell subsets. J. Immunol. 180(6), 3739-3745 (2008).

73 Takeda K, Oshima H, Hayakawa Y et al. CD27-mediated activation of murine NK cells. J. Immunol. 164(4), 1741-1745 (2000).

74 Sakanishi T, Yagita H. Anti-tumor effects of depleting and non-depleting anti-CD27 monoclonal antibodies in immunecompetent mice. Biochem. Biophys. Res. Commun. 393(4), 829-835 (2010).

75 Kelly JM, Darcy PK, Markby JL et al. Induction of tumorspecific $\mathrm{T}$ cell memory by NK cell-mediated tumor rejection. Nat. Immunol. 3(1), 83-90 (2002).

76 French RR, Taraban VY, Crowther GR et al. Eradication of lymphoma by CD8 $\mathrm{T}$ cells following anti-CD 40 monoclonal antibody therapy is critically dependent on CD27 costimulation. Blood 109(11), 4810-4815 (2007).
77 Vitale LA, He L-Z, Thomas LJ et al. Development of a human monoclonal antibody for potential therapy of CD27-expressing lymphoma and leukemia. Clin. Cancer Res. 18(14), 3812-3821 (2012).

- Analysis of the properties of the anti-CD27 monoclonal antibody $(\mathrm{mAb})$ that is currently in clinical trials.

78 He L-Z, Prostak N, Thomas LJ et al. Agonist anti-human CD27 monoclonal antibody induces $\mathrm{T}$ cell activation and tumor immunity in human CD27-transgenic mice. J. Immunol. 191(8), 4174-4183 (2013).

- Analysis of the mechanism of action of the anti-CD27 mAb that is currently in clinical trials.

79 Hünig T. The storm has cleared: lessons from the CD28 superagonist TGN1412 trial. Nat. Rev. Immunol. 12(5), 317-318 (2012).

80 Van Montfrans JM, Hoepelman AIM, Otto S et al. CD27 deficiency is associated with combined immunodeficiency and persistent symptomatic EBV viremia. J. Allergy Clin. Immunol. 129(3), 787-793.e6 (2012).

81 Salzer E, Daschkey S, Choo S et al. Combined immunodeficiency with life-threatening EBV-associated lymphoproliferative disorder in patients lacking functional CD27. Haematologica 98(3), 473-478 (2013).

82 Ansell SM, Northfelt DW, Flinn I et al. Phase I evaluation of an agonist anti-CD27 human antibody (CDX-1127) in patients with advanced hematologic malignancies. J. Clin. Oncol. 32(5), Suppl. abstract 3024 (2014).

83 Neyns B, Wilgenhof S, Van Nuffel AMT et al. A phase I clinical trial on the combined intravenous (IV) and intradermal (ID) administration of autologous TriMixDC cellular therapy in patients with pretreated melanoma (TriMixIDIV). In: ASCO Annual Meeting Proceedings 2519 (2011).

84 Rosenberg SA, Yang JC, Sherry RM et al. Durable complete responses in heavily pretreated patients with metastatic melanoma using T-cell transfer immunotherapy. Clin. Cancer Res. 17(13), 4550-4557 (2011). 